Recepción: 09/06/2018

Evaluación: $18 / 07 / 2018$

Aprobación: 18/09/2018

Artículo de Investigación-Científica

DOI: https://doi.org/10.22267/rhec.192222.51

\title{
Aproximación a las pedagogías alternativas: de la pedagogía de la diversidad a las pedagogías de la resiliencia en el marco del postacuerdo ${ }^{1}$
}

\author{
José Pascual Mora García ${ }^{2}$ \\ Universidad de Los Andes, Venezuela \\ Universidad de Cundinamarca, Colombia
}

\section{Resumen}

El artículo aborda las Pedagogías Alternativas como soporte epistemológico de la Línea de Investigación Pedagogías, Paz y Poblaciones Resilientes, en el marco del Postacuerdo de la región andina Cundiboyacense. Esta línea de investigación se macera en el Doctorado en Ciencias de la Educación, en el CADE, de la Universidad Pedagógica y Tecnológica de Colombia (UPTC-Tunja) y el emergente programa de Doctorado en la Universidad de Cundinamarca (U de C- Fusagasugá). Las categorías centrales que se abordan son: Pedagogías, Paz y Resiliencia. Desde el punto de vista metodológico se retoma la Historia de las mentalidades para su acercamiento a la investigación social. En ese sentido, resultado de la investigación se propone a la comunidad científica que entre las Pedagogías Alternativas, se incorpore el desarrollo de la Pedagogía Geomental, para el estudio de los andamios mentales resilientes en el tiempo estructural, a fin de superar las patologías sociales que inducen a la mentalidad de la violencia.

$1 \quad$ Este artículo se deriva de la investigación, en Postdoctorado, en la Universidad Pedagógica y Tecnológica de Colombia (UPTC), Tunja, bajo la dirección de la Dra. Diana Soto Arango (2018-2019).

2 Profesor Titular Emérito de la Universidad de Los Andes - Táchira, Venezuela. Filósofo (UCV, Caracas. Magíster en Gerencia Educativa (UNET). Diploma de Estudios Avanzados, DEA (Universidad Rovira i Virgili (URV), Tarragona, España. Doctor en Innovación y Sistema Educativo (URV), Tarragona, España. Doctor en Historia Económica y Social, (USM), Caracas, Venezuela. Grupo Hedure/Hisula UPTC, Past Presidente Red de Historia Latinoamericana SHELA. Presidente del IEH Nodo San Cristóbal. Investigador categoría Asociado (I) de Colciencias (convocatoria 2019) adscrito al Grupo Hisula-UPTC, Tunja. Investigador vinculado al Doctorado en Ciencias de la Educación de la Universidad de Cundinamarca. Correo electrónico: pascualmoraster@gmail.com. Código ORCID: https://orcid.org/0000-0002-5345-6808 
Respecto a la categoría Postacuerdo, en el sentido del Acuerdo final para la Terminación del Conflicto y la Construcción de una Paz estable y duradera. Igualmente, las Pedagogías Alternativas se inspiran en la diversidad epistemológica, entre las cuales se destacan, las Epistemologías del Sur, el Paradigma emergente al interno de la Sociedad de Historia de la Educación Latinoamericana (Shela) (Mora-García, Soto-Arango y Lima Jardilino (2017), las Pedagogías indígenas, entre otras. Se propone finamente, delinear el quiebre de las alcabalas que en el pasado llevaron a la intelectualización del saber pedagógico divorciado de las prácticas sociales; la mirada a la Paz, que se ofrece, es una paz con sentido decolonial, emancipador y duradero.

Palabras clave: Educación Alternativa; Educación y Cultura; Educación para la Paz; Investigación Pedagógica. 


\title{
An insight on alternative pedagogies: from the diversity pedagogy to the resilient pedagogies within the post-conflict process
}

\begin{abstract}
This article analyzes alternative pedagogies as an epistemological support for the line of research Pedagogies, peace and resilient populations, within the framework of the Post-erection of the Cundiboyacean Andean Region. The Doctorate in Education Sciences, in CADE, of the Universidad Pedagógica y Tecnológica of Colombia (UPTC-Tunja) and the emerging PhD program at the Universidad de Cundinamarca (U. de C.- Fusagasugá) develop this line of research. The central categories addressed are: Pedagogies, Peace and Resilience. From the methodological point of view, the History of Mentalities is taken up again in order to approach the social research. In this sense, and as a result of the research process, it is recommended that the Geomental Pedagogy will be incorporated as part of Alternative Pedagogies, for the study of resilient mental scaffolds in structural time, in order to overcome the social pathologies that induce the mentality of violence.
\end{abstract}

Regarding the Post-Conflict category, in the sense of the Final Agreement for the end of the conflict and the construction of a stable and lasting peace, the Alternative Pedagogies are inspired by epistemological diversity, among which the Epistemologies of the South, the Emerging Paradigm within the Latin American Education History Society (Laehs) (Mora-García, Soto-Arango and Lima Jardilino (2017), and the Indigenous Pedagogies stand out. It is also recommended to delineate the break of the taxes that in the past led to the intellectualization of pedagogical knowledge divorced from social practices; the look at Peace, which is offered, is a peace with a decolonial, emancipatory and lasting sense.

Keywords: Alternative Education; Education and Culture; Education for Peace; Pedagogical Research. 


\section{Abordagem das pedagogias alternativas: da pedagogia da diversidade às pedagogias de resiliência no âmbito do Pós-Acordo}

\section{Resumo}

O artigo trata das Pedagogias Alternativas como suporte epistemológico das Pedagogias da Linha de Pesquisa, Paz e Populações Resilientes no âmbito do Pós-Acordo da região andina de Cundiboyacense. Essa linha de pesquisa é macerada no Doutorado em Ciências da Educação no Cade da Universidade Pedagógica e Tecnológica da Colômbia (UPTC-Tunja) e no emergente programa de doutorado na Universidade de Cundinamarca (U de C-Fusagasugá). As categorias centrais abordadas são: Pedagogias, Paz e Resiliência. Do ponto de vista metodológico, a História das mentalidades é retomada por sua abordagem à pesquisa social. Nesse sentido, como resultado da pesquisa, propõe-se à comunidade científica que entre as pedagogías alternativas seja incorporada o desenvolvimento da pedagogiaa Geomental, para o estudo de estruturas mentais resilientes no tempo estrutural, a fim de superar as patologias sociais que induzem à mentalidade da violência.

Em relação à categoria Pós-Contrato, no sentido do acordo Final para o Término do Conflito e a Construção de uma Paz Estável e Duradoura. Da mesma forma, as pedagogias alternativas são inspiradas na diversidade epistemológica, entre as quais as mais importantes as Epistemologias do Sul, o Paradigma emergente na parte interna da Sociedade da História da Educação Latino-Americana (Shela) (Mora-García, Soto-Arango e Lima Jardilino (2017), As Pedagogias indígenas, entre outras, é finamente proposto para delinear o colapso das alcabalas que no passado levaram à intelectualização do conhecimento pedagógico separado das práticas sociais; o olhar para a Paz, oferecido, é uma paz com um sentido decolonial, emancipatório e duradouro.

Palavras-chave: Educação Alternativa; Educação e Cultura; Educação para a Paz; Pesquisa Pedagógica. 


\section{Introducción}

Este trabajo es un estudio que expone preliminarmente los resultados del Postdoctorado en Historia de la Educación, en el CADE, de la Universidad Pedagógica y Tecnológica de Colombia (UPTC), bajo la dirección de la Dra. Diana Soto Arango. Uno de los logros fue la consolidación de la Línea de investigación: Pedagogías, Paz y Poblaciones Resilientes, en el CADE UPTC-Tunja, y la creación de la misma línea de investigación como soporte epistemológico en el proyecto de Doctorado en Ciencias de la Educación en la Universidad de Cundinamarca, Fusagasugá.

En este trabajo, por un lado, se presentan algunos de los fundamentos epistemológicos acerca de las Pedagogías, la Paz y la Resiliencia y, por otro, como un ejercicio de contextualización, se da cuenta del campo intelectual en el marco del Postacuerdo, en la región geohistórica cundiboyacense.

La maduración de la línea de investigación tiene más de 10 años, en los cuales se pueden observar los aportes a la comprensión de las Pedagogías, la Paz, y la Resiliencia. En este sentido, se presenta el desarrollo de las Pedagogías Alternativas, que toman como referencia el compromiso que exige la sociedad a la universidad respecto al tema de la Paz. El entronque entre las Pedagogías y la Investigación social es necesario, no solo para idealizar el objeto pedagógico, sino para aplicar la tasa de retorno social que requiere todo proyecto educativo. Lo importante es romper los cercados que en el pasado llevaron a una intelectualización del saber pedagógico divorciado de las prácticas sociales; en este trabajo, compartimos, con Marc Bloch, que "antes de ser historiadores de tal o cual rama, somos simplemente historiadores (...) en esto nos alineamos de buena gana con (...) los destructores de cercados (...) No existe en el mundo obra completa alguna. Lo esencial es abrir caminos. Los Annales mientras vivan (...) continuarán luchando contra la nefasta compartimentación de las ciencias.”3

\section{Las Pedagogías Alternativas}

La comprensión de la Paz y el empoderamiento de las Poblaciones Resilientes en aras de la superación de la violencia estructural se inspira en las Pedagogías Alternativas, que sirven de soporte epistemológico a las prácticas pedagó-

3 Marc Bloch, La Historia rural francesa: caracteres originales (Barcelona: Crítica, 1978), 38. 
gicas no solo vinculadas a la escolaridad, sino, también, a la Pedagogía Social, y las reflexiones que desde la universidad colombiana ${ }^{4}$ aportan al tema de la paz ${ }^{5}$ a fin de desarrollar engramas cognitivos y la maceración de "andamios mentales" (Febvre) para una paz sostenible, duradera y emancipatoria. La universidad debe cumplir un rol fundamental en el empoderamiento de las comunidades: "Los aportes de la universidad pretenden ser una contribución que conlleva ir de la mano de las comunidades urbanas y rurales para transitar los derroteros que permitan alcanzar el proceso de construcción de paz. Lo anterior podrá hacerse realidad con el apoyo de las pedagogías alternativas (...), con su función de educar personas empoderadas y con argumentos para que, en conjunción con las experiencias de la población, participen de manera activa en la consecución de una vida mejor."6

Las Pedagogías Alternativas se abordan desde la Pedagogía de la Diversidad (Dos Santos, 2003), la Pedagogía de la Resiliencia, y otras pedagogías. La Pedagogía de la Diversidad ${ }^{7}$ (Dos Santos, 2003) ayuda a la comprensión de las sociedades multiculturales y pluriétnicas. Aun cuando los esfuerzos se han sostenido desde la Declaración Universal de Derechos Humanos (Unesco, 1948), en las últimas décadas ${ }^{8}$ la comunidad científica nacional e internacional ha realizado pronunciamientos cónsonos con esta necesidad:

En la década de 1990, innumerables movimientos realizados en distintos países del mundo, como el movimiento de Educación para Todos, meta de la Conferencia de Jomtien, en Tailandia, se unió a otra serie de acciones defendidas en las Conferencias sobre Desarrollo Humano en Copenhague; sobre Desarrollo de la Mujer en Beijing; en la Conferencia de Hamburgo sobre Educación del Joven Adulto

$4 \quad$ Celina Trimiño Velásquez y Luisa Amézquita Aguirre, "Reflexiones desde la universidad sobre educación en derechos humanos y para la paz", Revista Historia de la Educación Latinoamericana. Vol. 20. No. 31 (2018): 101-124.

$5 \quad$ José Ignacio Bolaños Motta, "Aulas multiculturales y aulas de paz. Dos propuestas para un país en Postconflicto", Revista Historia de la Educación Latinoamericana. Vol. 20. No. 31 (2018): 83-100.

6 TrimiñoVelásquez y Amézquita Aguirre, "Reflexiones desde...", 103.

7 Maria Dos Santos, Pedagogía de la diversidad (Santiago de Chile: LOM, 2003).

8 En los Lineamientos de Política para la Atención Educativa a Poblaciones Vulnerables (julio de 2005), el Ministerio de Educación de Colombia reconoce la diversidad y heterogeneidad de las regiones en el marco de la descentralización; en el año 2007, con la Declaración de Educación para Todos: Un asunto de Derechos Humanos (Unesco, 2007); en el 2008, la Conferencia Internacional sobre Educación (Ginebra, Suiza), denominada Educación inclusiva, asume la inclusión como un proceso de respuesta a la diversidad; la Constitución Política de Colombia, de 1991, y la Ley General de Educación (Ley 115, 1994) promueven las condiciones de igualdad y equidad en la educación colombiana, entre otras. 
Trabajador y en la Declaración de Salamanca, como ya nos referimos, en el sentido de buscar acciones que apunten para el reconocimiento de la necesidad de convivir con las diferencias entre los seres humanos una vez que el mundo contemporáneo tiene en la pluralidad sus fundamentos y organización. La Declaración Mundial de Educación para Todos, particularmente, pone en duda los procesos escolares tradicionales, intensificando las reflexiones en torno a cómo hacer operativo el principio democrático de Educación para Todos, por medio de la modernización de los recursos, del acceso al aprendizaje de todos los alumnos, del perfeccionamiento de los mecanismos de gestión educacional y de la práctica pedagógica de los profesores. ${ }^{9}$

La Constitución de 1991, también, abrió un capítulo para el tema intercultural. En 1991, se aprobó en Colombia una nueva Constitución, que reemplazó a la antigua, del año 1886. A diferencia de la anterior Constitución, caracterizada por una comprensión del Estado anclado en la tradición hispánica, católica y castellana, la de 1991 considera, en el Artículo 7, una nación pluriétnica y pluricultural: "El Estado reconoce y protege la diversidad étnica y cultural de la Nación Colombiana." Colombia abrió un compás, en este sentido, para apostar a la Paz.

El Acuerdo Final de Paz y las implicaciones del postacuerdo requieren de un tratamiento especial para el manejo de la Paz, razón por cual se necesita la comprensión de las Pedagogías a la luz de los paradigmas pedagógicos emergentes; sin un proceso formativo que logre llegar a todos los sectores de la sociedad, será imposible la Paz. No son suficientes los Acuerdos firmados; se requiere de la Repedagogización social, para impactar en el inconsciente colectivo. Se trata de proponer y consolidar estrategias de repedagogización social, en aras de formar a los docentes, líderes sociales, personal gubernamental y no gubernamental, así como el personal de las ONG's humanitarias, para trabajar por una paz sostenible.

Desde el punto de vista de los paradigmas emergentes, se comprende a la Paz desde las Pedagogías del Sur, y el Paradigma emergente de la Historia de la educación latinoamericana (Shela). ${ }^{10}$ Asumimos el concepto de resiliencia, con Suárez (2004): "'en la esfera de las ciencias sociales, la incorporación de la noción de resiliencia se empezó a expresar principalmente desde la psicolo-

\footnotetext{
9 Dos Santos, "Pedagogía", 2.

10 José Pascual Mora García, "Paradigma historiográfico emergente en la revista de historia de la educación latinoamericana (Rhela)", Pueblo-Continente, Vol. 25, No. 1 (2014), 295-312.

11 Al respecto, puede verse Elbio Néstor Suárez Ojeda, "Introducción. Resiliencia y Subjetividad", en Resiliencia y Subjetividad. Los ciclos de la vida. Tramas sociales, comps. Aldo Melillo; Elbio Néstor Suárez Ojeda y Daniel Rodríguez (Buenos Aires, Paidós, 2004).
} 
gía; principalmente para referirse a las respuestas "positivas" de los individuos que se enfrentaron ante un acto perturbador o adverso. La noción de resiliencia toma auge primero desde los países anglosajones, como Inglaterra (Rutter, 1993) y Estados Unidos de Norteamérica (Werner, 1994) y, luego, se propaga a los otros países de Europa occidental (Francia, Países Bajos, Alemania y España), para, finalmente, aterrizar en América Latina." "2 Sin embargo, reconocemos en los antecedentes epistemológicos los siguientes planteamientos del origen del concepto de resiliencia: Holling (1973); ${ }^{13}$ Nadia P. Abesamis, Colleen Corrigan, Mark Drew, Stuart Campbell, Giselle Samonte (2006). ${ }^{14}$ En el contexto latinoamericano, se retoman los paradigmas situados desde autores como Leopoldo Zea, Paulo Freire, ${ }^{15}$ Hugo Zemelman, ${ }^{16}$ Enrique Dussel, ${ }^{17}$ Luis Bigott; ${ }^{18}$ los enfoques delineados desde los centros de investigación, como Clacso, ${ }^{19}$ o los grupos de investigación, como es el caso del Grupo Hisula-UPTC, ${ }^{20}$ e investigaciones rea-

12 Suárez Ojeda, "Introducción. Resiliencia y Subjetividad"

13 C. S. Holling, "Resilience and stability of ecological systems", en Annual Review of Ecology and Systematics. Vol. 4 (1973), 1-23; C. S. Holling y B. H. Walker (2003), "Resilience defined", en Internet Encyclopedia of Ecological Economics, ed. International Society of Ecological Economics, http://www.ecoeco.org/pdf/resi lience.pdf

14 Nadia P. Abesamis et al., Social Resilience: A literature review on building resilience into human marine communities in and around MPA networks, MPA Networks Learning Partnership, Global Conservation Program, USAID (septiembre 2006), http://www.reefresilience. org/pdf/Social_Resilience_Literature_Review.pdf

15 Paulo Freire, Pedagogía de la tolerancia (México: CREFAL, 2006)

16 Periódico, U. (1). "Con el maestro Hugo Zemelman en la Universidad de Nariño - Entrevista", Revista Historia de la Educación Colombiana. Vol. 17. No. 17 (2014), 19-27, http://revistas. udenar.edu.co/index.php/rhec/article/ view/2110; Hugo Zemelman y Estela B. Quintar, Conversaciones acerca de interculturalidad y conocimiento (México: IPN/IPECAL, 2007).

17 Enrique Dussel, "La introducción de la transformación de la filosofía de K.-O. Apel y la filosofía de la liberación. Reflexiones desde una perspectiva latinoamericana", en Fundamentación de la ética y la filosofía de la liberación, Karl-Otto Apel et al. (México: Siglo XXI, 1992), 45-104.

18 José Pascual Mora García, "Luis Bigott y la contribución a la pedagogía contrahegemónica", Heurística. No. 19 (2016), 310-322. Conferencia enmarcada en el Proyecto de investigación: La UPTC en los desafíos del siglo XXI, una prospectiva desde los estudiantes y docentes GI 165 del Grupo HISULA, UPTC, coordinado por la Dra. Diana Soto Arango, en el que participo como Investigador externo. VI Congreso internacional sobre historia y prospectiva de las universidades de Europa y América (CIHPUEA). Tunja, Boyacá, 10-12 de octubre de 2016.

19 Norma Giarracca, comp., ¿Una nueva ruralidad en América latina? (Buenos Aires: Clacso, 2000).

20 José Pascual Mora García, "Las Pedagogías de la Resiliencia, estudio de casos", Conferencia en la Cátedra Historia y Prospectiva de la Universidad Latinoamericana (HISULA Cade UPTC), Tunja, 26 de agosto de 2018, http://www.uptc.edu.co/export/sites/default/facultades/f_educacion/doctorado/educacion/newsletter/doc/2018/newsletter_1_2018.pdf 
lizadas sobre resiliencia; entre ellas: Liliana Paternina; ${ }^{21}$ y Victoria Fontan, ${ }^{22}$ que aportan sus análisis sobre las relaciones entre familia, paz y resiliencia. Además, resulta muy relevante tomar en cuenta los aportes desde instancias internacionales, como ONU, el acompañamiento de FAO en los programas que han proyectado, para Colombia, la Resiliencia como estrategia:

(...) tras más de cincuenta años de conflicto armado interno, Colombia vive un momento de esperanza después de la firma del Acuerdo de Paz en noviembre de 2016 entre el gobierno colombiano y la guerrilla de las Fuerzas Armadas Revolucionarias-Ejército del Pueblo (FARC-EP). En este contexto, la Representación de la FAO en Colombia ha elaborado un "Estrategia de Acompañamiento de FAO a la Implementación del Acuerdo de Paz en Colombia" que define las líneas de trabajo y concreta lo establecido en el Marco Programático de País (MPP) 2015-2019. El programa de resiliencia se inserta en estos marcos poniendo énfasis en los aspectos de construcción de resiliencia como concepto vertebrador fundamental en los escenarios de posconflicto... ${ }^{23}$

Este estudio busca deconstruir los paradigmas pedagógicos hegemónicos que alienan al ser social en el desarrollo de una Paz sostenible. En este sentido, se deconstruye desde el paradigma patriarcal hasta los paradigmas que imponen la unanimidad de pensamiento y potencian la dialéctica de la negación. Desde la metodología de la Historia de las mentalidades, se aborda el entronque entre los Andamios geomentales (Mora-García) y Pedagogía, a fin de advertir lo extraordinariamente productivo que resulta efectuar una lectura transversal de las estructuras mentales de la sociedad en la comprensión del tema pedagógico, porque la Pedagogía no se reduce exclusivamente a la escuela, sino, al intervenirla, como Aparato Ideológico del Estado, requiere de una comprensión crítica de los

$21 \quad$ Cfr. Liliana Paternina Soto, "El texto escolar sustentado en la resiliencia para poblaciones vulnerables. Fusagasugá 2008-2011”, Rhela. No. 16 (2011); J. Klevens et al., Epidemiología de la delincuencia en cinco ciudades. [Epidemiology of Delinquency in Five Cities], (Bogotá: Technical report to Colciencias, 1997); Joanne Klevens y Juanita Roca, "Nonviolent Youth in a Violent Society: Resilience and Vulnerability in the Country of Colombia", Violence and Victims. No. 14 (1999), 311-322.

22 Victoria Fontan, Relación entre "familia, resiliencia y paz" en el contexto colombiano" (audio), https://www. javerianacali.edu.co/relacion-entre-familia-resiliencia-y-paz-en-el-contexto-colombiano; Victoria Fontan se formó en Estudios de Paz y Desarrollo, en la Universidad de Limerick, Irlanda; es Doctora en Educación, especialidad Mediación Pedagógica, en la Universidad de La Salle, Costa Rica.

23 Organización de las Naciones Unidas para la Alimentación y la Agricultura (FAO), “Colombia: Programa de resiliencia (2017-2020)", http://www.fao.org/emergencies/recursos/ documentos/recursos-detalle/es/c/903122/ 
procesos. El docente o el pedagogo social es un actor que crea teoría y produce ideología; por eso, apostamos por una pedagogía social emancipatoria (Habermas) y liberadora (Freire, Dussel).

\section{La Pedagogía Geomental, un enfoque desde la Historia de las mentalidades a las Pedagogías Alternativas}

La pedagogía ha evolucionado como saber pedagógico divorciado de la capacidad de conectarse con los saberes resilientes de la cultura de resistencia que anidan en los andamios mentales. Aquí está la premisa central de este capítulo. Desde el punto de vista metodológico, buscamos integrar esos dos discursos: Pedagogías y Andamios mentales, en el tiempo de longue durée o tiempo estructural. Retomamos, de la tradición historiográfica de la Escuela de Annales, el concepto de mentalidad (Bloch), para integrar lo geohistórico y las mentalidades, lo que hemos denominado Andamios geomentales (Mora-García, 2001). Las pedagogías de la resistencia, entre las que destacamos la resiliencia, la diversidad, la paz, las pedagogías del Sur, y los DDHH, tienen sus fundamentos en los andamios mentales de nuestros pueblos, en que se entroncan los elementos del capital social (Bourdieu) y que funcionan como capital simbólico, que reproducen los valores de esa población resiliente. Integramos lo Geomental a las Pedagogías de la Resiliencia, como un aporte de nuestras reflexiones para la Paz en el marco del Postconflicto. No es un estudio acabado, sino un estudio en proceso, en el que se señala una propedéutica a seguir en la búsqueda de la Paz en Colombia.

La pedagogía, desde cuando surgió como ciencia, con Juan Amos Comenio, buscó la construcción de un saber pampédico; es decir, el "saber todo de todo" que, poco a poco, se ha transformado en un saber hiperespecializado, y terminado por ser un saber para la obediencia (la enseñanza doctrinaria catequística), la domesticación a través del pensamiento instrumental, la subyugación, la sujeción y la adaptación a la sociedad (Durkheim). La pedagogía expuesta para vencer la adversidad y la construcción de un saber decolonia ${ }^{24}$ se instaura siste-

$24 \quad$ Catherine Walsh, ed., Pedagogías decoloniales, prácticas insurgentes de resistir, (re)existir, y (re)vivir, Tomo I (Quito: Abya-Yala, 2013). 
máticamente desde la obra de Simón Rodríguez ${ }^{25}$ y los pensadores que lograron una visión prospectiva desde el siglo XIX. La herencia de la pedagogía decolonial tiene sus raíces en los saberes indígenas prehispánicos:

el enlace de lo pedagógico y lo decolonial inicia con la invasión colonial-imperial, es con el establecimiento de los virreinatos hispánicos en los territorios mesoamericanos y andinos que empieza a manifestarse y tomar forma. De hecho, en los manuscritos del Huarochirí y del Popul Vuh, compilados por escribanos indígenas en el siglo XVI en lo que hoy se conoce como Perú y Guatemala respectivamente, podemos atestiguar estrategias que subvierten la lógica civilizatoria colonial a la vez que sitúan cosmologías propias, haciendo pensar el mundo, la creación, la relación entre seres — vivos y muertos, humanos y no humanos - y con la naturaleza de modo para nada occidental. ${ }^{26}$

Las pedagogías indígenas han encontrado en estos saberes la posibilidad de recuperar sus imaginarios; en su resiliencia, podemos pensar la posibilidad de una pedagogía que se reencuentre con su ser y saber ancestral, o sus andamios geomentales. El grupo Hisula-UPTC ha desarrollado adelantos importantes en esta dirección, como es el caso de Diego Naranjo Patiño, quien realizó un proyecto sobre el Resguardo Unido U'wa, en Boyacá, pero, en Colombia, la Comunidad Científica ha realizado avances muy importantes, en materia de Derechos Huma$\operatorname{nos}^{27}$ y las políticas lingüísticas, ${ }^{28}$ al crear conciencia sobre la recuperación de la memoria histórica lingüística en el tiempo de larga duración. Igualmente, reseñamos los trabajos de Armando Suescún, sobre la recuperación de las raíces muiscas en el Altiplano cundi-boyacense. ${ }^{29}$ En la reconstrucción geográfica muisca, resaltamos a Carmenza Olano Correa, quien presenta un estudio sobre el Resguardo. ${ }^{30}$

25 José Pascual Mora García, (2019) "Simón Rodríguez, precursor del pensamiento pedagógico decolonial", Conferencia en el Doctorado de Educación, Universidad Federal de Ouro Preto, Brasil, 5 de junio de 2019, https://www.youtube.com/watch?v=10IVNJtX9W0

26 Walsh, "Pedagogías decoloniales", 33.

27 El concepto de Derecho Humano Lingüístico lo han incorporado Tove Skutnabb-Kangas y Robert Phillipson, en 1994. Cfr. Tove Skutnabb-Kangas y Robert Phillipson, "Language Rights: Principles, Enactment, Application", en Language Rights. Vol. 1, Tove Skutnabb-Kangas et al,, eds. (Londres/Nueva York: De Gruyter Mouton, 2017), 28-67.

28 Roberto Pineda Camacho, El derecho a la lengua: una historia de la política lingüística en Colombia (Bogotá: Uniandes, 2000).

29 Armando Suescún, "El Derecho chibcha, siglo IX-siglo XVI", en Derecho y sociedad en la Historia de Colombia, Tomo I, $2^{\mathrm{a}}$ ed. (Tunja: UPTC, 2012).

30 Carmen Olano Correa, Espacios Amerindios: mhuyscas y chibchas de Boyacá, tierras de resguardo, siglos XVI al XVIII (Bogotá: Centro Desarrollo Ideas, 2010). 
En la Maestría de DDHH, de la UPTC, ${ }^{31}$ encontramos trabajos resultado de las tesis de Maestría, que hemos publicado en el monográfico que organizamos para mostrar la presencia de los sujetos empoderados en las comunidades indígenas; en particular, citamos a Elena Oliveros Marín, "Relación entre derechos humanos y derechos lingüísticos en el pueblo indígena Kankuamo de la Sierra Nevada de Santa Marta". ${ }^{32}$ Igualmente, citamos otros antecedentes, ${ }^{33}$ como aquellos que apuestan por la educación inclusiva en el marco del Proceso de Paz; ${ }^{34} \mathrm{o}$ aquellos que plantean repedagogizar a los excombatientes con miras a la reinserción labora ${ }^{35} \mathrm{e}$ incluir a las personas con necesidades especiales en la educación y empoderamiento de los DDHH. ${ }^{36}$ El proceso de reinserción debe verse no solo como un proceso instrumental de quienes han estado fuera de la ley, sino como un proceso de repedagogización social global, porque no se trata de estigmatizar, sino de comprender; quizá, por eso, estamos más cerca de referirnos a una pedagogía de la comprensión, más que una pedagogía del conocimiento.

En nuestro caso, hemos desarrollado una línea de investigación que nos permitimos retomar para alimentar los lineamientos de la Pedagogía Indígena, a propósito del denominado Día de la Resistencia Indígena. El 12 octubre dejó de ser un día para la celebración del despojo, por eso se instituyó el día de la Resistencia Indígena, según Gaceta Oficial No 5.605, de 10 de octubre de 2002, emitido por la Presidencia de la República de Venezuela. Atrás quedó la concepción hispanista que potenciaba el eurocentrismo y la anglobalización; aquella que buscaba perpetuar la cultura occidental como La Cultura, cual cultura única, aquella que consideraba a la cultura anglo-europea como la cultura que debía

31 Nuestro trabajo como Profesor invitado a la Maestría de DDHH de la UPTC, en las sedes Tunja y Bogotá, entre el 2016-2019, nos ha permitido, además, formar parte como jurado internacional de más de 10 tesis de Maestría en DDHH.

32 Elena Oliveros Marín, "Relación entre derechos humanos y derechos lingüísticos en el pueblo indígena Kankuamo de la Sierra Nevada de Santa Marta”, Heurística. No. 20 (2017). Tesis de Maestría en DDHH, UPTC.

33 Claudia María Rojas Quiñónez, "La diversidad lingüística en Colombia: protección jurídica de las lenguas indígenas", Pensamiento Jurídico. No. 22 (2008), 253-292.

34 Nohora Isabel Manchego Bonilla, "Ajustes razonables y derechos humanos: una perspectiva de equidad, dignidad y trascendentalidad en la inclusión educativa", Heurística. No. 20 (2017), 28-40.

35 Andrea Delgado Martínez, "Oportunidades laborales para excombatientes: percepciones de los generadores de empleo en el Quindío y Risaralda", Heurística. No. 20 (2017).

36 Heidy Lorena Cabra García, "Barreras para la inclusión social de las personas con discapacidad, un escenario de derechos humanos", Heurística. No. 20 (2017). 
imponerse. Con Octavi Fullat, compartimos que la cultura occidental surgió a partir de la fusión de tres grandes bloques culturales: Grecia, Roma y la cultura judaica y, como tal, tiene dos mil años de imposición forzada. Para Occidente, las culturas alternas no existen, y esa fue la lógica que decretó la aniquilación de nuestras raíces pobladoras prehispánicas. Quizá por esa razón expresaba Arnaldo Esté $(1986)^{37}$ que NO SOMOS OCCIDENTALES.

La lengua y el imperio van de la mano. Antonio de Nebrija señalaba a la reina Isabel que "siempre la lengua fue compañera del imperio, y de tal manera lo siguió, que juntamente comenzaron, crecieron y florecieron, y después junta fue la caída entre ambos. ${ }^{38}$ La castellanización del indígena fue el gran proyecto del imperio español, ejercicio que se hizo sin considerar la tradición de las lenguas autóctonas. ${ }^{39}$ Nos recuerda que la escuela fue el instrumento que utilizó el imperio para diseminar su lengua, no obstante que el nuevo código del Derecho Indiano prescribía que se debía respetar las lenguas autóctonas. También, muy a pesar de que el Concilio Provincial Mejicano, de 1585, y el Concilio Limense III, de 1583 , mandaran a que se enseñara en la lengua de los indios y, habría que agregar aquí, los esfuerzos realizados a favor de los indios por las órdenes franciscanas y dominicas en el Nuevo Mundo. Pero esta es la paradoja de la discrecionalidad legal ejercida por el imperio: "se acata, pero no se cumple." Fue así como entró, también, "el signo de la cruz en las empuñaduras de las espadas", metáfora excelentemente recreada por Eduardo Galeano. ${ }^{40}$ Las lenguas náhuatl, quechua, aimará y nuestros dialectos de raigambre muisca, chibcha, Tunebo y Bari, así como la antigua lengua Torondoy, se negaron y solo lograron mantenerse algunas ocultamente como cultura de resistencia. Precursores de estos estudios etno-lingüísticos indígenas, en la región andina venezolana, fueron José Ignacio Lares, Tulio Febres Cordero, Alfredo Jahn y Julio César Salas, pero los estudios realizados por Jacqueline Clarac de Briceño, Gladys Gordónes, Lino Meneses, Omar González Ñáñez, Reina Durán y Luis Bastidas, de la Universidad de los Andes y Museo Antropológico de Mérida, han permitido ir más allá, para anunciar que teníamos un conjunto de lenguas, que pueden reagruparse bajo el calificativo de lenguas "chontales", cuyo imaginario se está rescatando.

\footnotetext{
37 Arnaldo Esté, Los maleducados: de la Escuela Básica a la Universidad (Caracas: UCV, 1986).

38 Antonio de Nebrija, Gramática de la Lengua Castellana (Salamanca: s/e, 1492).

39 Silvio Savala, Filosofía de la conquista y otros textos (Caracas: Biblioteca de Ayacucho, 2005).

40 Eduardo Galeano, Las venas abiertas de América Latina (70ª ed., México: Siglo XXI, 2004).
} 
El filósofo mexicano Leopoldo Zea nos invita a la construcción de filosofía de la resistencia desde América Latina, una filosofía de la resistencia indígena y de la negritud; ${ }^{41}$ por eso, afirma:

negritud e indigenismo son conceptos ideológicos (...) el hombre blanco ha hecho de su blanquitud una abstracción de lo humano en la que sólo él tiene cabida. El hombre de África y el hombre de América Latina, por el contrario, harán de lo que les distingue racial y culturalmente de otros hombres, el punto de partida de su semejanza con ellos. ${ }^{42}$

Es la propuesta de la síntesis en lo mestizado; somos una suerte de síntesis dialéctica en la sangre; somos lo uno en lo mestizado versus las supuestas razas puras. Por eso, el cartesianismo, que matematizó nuestra cotidianidad, no nos dice nada en América Latina; somos más Pathos (sentimiento) que Logos (Razón). Y eso nos salva. Somos más hombres del sentimiento que de la fría Razón; nuestras musas cantan una oda al "Sentio, ergo sum", esto es, al "Yo siento, luego existo", diría Leonardo Boff. ${ }^{43}$ Nuestro pensamiento contiene una lógica polivalente, en la que el "realismo mágico" y la Razón se entrecruzan. No estamos sujetos irremediablemente al principio de no contradicción aristotélico; en América Latina, cabe A y no A al mismo tiempo, para decirlo con lenguaje silogístico. Nuestro imaginario lo conforman manifestaciones monoteístas y manifestaciones ancestrales-protorreligiosas politeístas; de Bolívar a Changó, todos tienen un espacio en la imaginería criolla. En el altar de nuestras casas están Jesucristo, la Virgen María, junto a la Santísima Trinidad, pero, también, están María Lionza, el negro Miguel, Guaicaipuro y las cortes criollas. Esos somos los venezolanos y latinoamericanos; como dijera Augusto Salazar Bondy, somos una yuxtaposición cultural, y no pidamos que se minen nuestros imaginarios.

En nuestras culturas indígenas, se destruyeron los símbolos de sus imaginarios para imponer la cruz y la espada; esa generación abortada requiere ser emancipada en su cultura de resistencia para la modernidad alternativa. La lógica occidental elaboró un cartabón para adiestrarnos en el culto a la Razón y el odio a la imaginación. Solo el Romanticismo, el Surrealismo y el Simbolismo fueron los bastiones de resistencia de los valores de lo imaginario frente al cientificismo racionalista y empirista.

\footnotetext{
41 José Pascual Mora-García, "Leopoldo Zea, el filósofo de la negritud y el indigenismo", Revista de Ciencias Sociales de la región Centroccidental. No 9 (2004), 163-171.

42 Leopoldo Zea, Dialéctica de la conciencia americana (México: UNAM, 1974), 57.

43 Leonardo Boff, San Francisco de Asis: Ternura y vigor (Caracas: Ediciones Paulinas, 2002).
} 
Desde Freud, sabemos que el pensamiento no trabaja solo a pleno día, que en las profundidades de la noche y las experiencias tenebrosas del inconsciente determinan el mundo de la vida racional. Todo pensamiento humano es representación; es decir, pasa por articulaciones simbólicas. Lo imaginario es el conector de toda representación humana. Lo heroico y lo místico, lo dramático y lo real forman parte de un mismo ser. Por eso, tanto el alma como el pensamiento son "atigrados"; la unanimidad de opinión es simplista. Se trata, entonces, de recrear los imaginarios y desnudar la Razón, porque son la base de nuestra memoria colectiva; por tanto, "se debe actuar de modo que la memoria colectiva sirva a la liberación, y no a la servidumbre de los hombres." ${ }^{44}$

Los engramas de las Pedagogías indigenistas emergentes encuentran en las Pedagogías decoloniales un aliado para poder expresar su potencialidad y, en consecuencia, son herramientas para las Pedagogías de la resiliencia, porque son saberes que

no están pensados(as) en el sentido instrumentalista de la enseñanza y transmisión del saber, tampoco están limitadas al campo de la educación o los espacios escolarizados. Más bien, y como dijo una vez Paulo Freire, la pedagogía se entiende como metodología imprescindible dentro de y para las luchas sociales, políticas, ontológicas y epistémicas de liberación. Las luchas sociales también son escenarios pedagógicos donde los participantes ejercen sus pedagogías de aprendizaje, desaprendizaje, reaprendizaje, reflexión y acción. ${ }^{45}$

Por eso, asumimos la pedagogía en el sentido plural ${ }^{46}$, porque se trata de

Pedagogías entendidas de manera múltiple: como algo dado y revelado; [que lleva a] abrir paso, traspasar, interrumpir, desplazar e invertir prácticas y conceptos heredados, estas metodologías síquicas, analíticas y organizacionales que usamos para saber lo que creemos que sabemos para hacer posible conversaciones y solidaridades diferentes; como proyecto tanto epistémico como ontológico ligado a nuestro ser. ${ }^{47}$

\footnotetext{
44 Jacques Le Goff, El orden de la memoria (Barcelona: Paidós, 1991), 183.

45 Walsh, ed., "Pedagogías decoloniales", 29.

46 M. Jaqui Alexander, Pedagogies of Crossing. Meditations on Feminism, Sexual Politics, Memory, and the Sacred (Durham, NC: Duke University Press. 2005), 7, citada por Walsh, Pedagogías decoloniales..., 30.

47 Jaqui Alexander, Pedagogies of Crossing, 29.
} 
En nuestro caso, nos proponemos presentar el entronque entre resiliencia y estructuras geomentales, para mostrar que, en el zócalo de la memoria colectiva, se encuentra el capital social que posibilita la historia de la resiliencia de los pueblos. Como consecuencia de ello, surgen múltiples definiciones sobre lo que es resiliencia. Se requiere integrar todas las miradas posibles. En este sentido, la resiliencia puede considerarse como un potencial que tienen las comunidades para vencer las dificultades en un contexto de adversidad significativa, pero, sobre todo, buscamos conectar la resiliencia con los andamios mentales, por ser el reservorio donde se fecundan las manifestaciones culturales y valores que tienen las comunidades y se anida la base de una resiliencia colectiva.

En este punto, la Resiliencia y los andamios mentales se conectan. En América Latina, con una raíz de historia violentada, es determinante la recuperación de la Pedagogía decolonial, para poder encontrar la capacidad de superación de la mentalidad de la violencia; sobre la violencia se ha ejercido más violencia y, así, se instauró una violencia histórica, que tiene sus raíces en la violencia estructural. Desde la llegada del pensamiento eurocentrista, comenzaron las clases para imponer esa pedagogía de la dominación que coexisten en los andamios mentales; esas pedagogías anidaron los engramas cognitivos para la domesticación.

En este sentido, Stephen Nathan Haymes señala la presencia de una pedagogía esclava, que germinó en sus andamios mentales para escapar a la represión:

(...) como pueblo con esperanza, usaron sus posibilidades para minimizar y rehusar el control que las realidades y rutinas, que se tomaban como verdaderas, tenían sobre su imaginación. Esta imaginación pedagógica les permitía mitigar y resistir las formas en que la aflicción destroza al alma. Mediante el trabajo simbólico —es decir, mediante sus canciones, cuentos y rituales de confirmación, por ejemplolos esclavos se enseñaban a sí mismos el significado moral y ético de crear un sentimiento de pertenencia comunitaria. Al forjar una comunidad de pertenencia, la cultura del esclavo funcionaba pedagógicamente para convertir a los esclavos en seres humanos, mediante la mitigación de su sufrimiento. ${ }^{48}$

$48 \quad$ Stephen Nathan Haymes, Race, Pedagogy and Paulo Freire. En Conferencia Internacional A reparação e a descolonização do conhecimento. Memorias (Salvador, Bahía: UFBA/Atitude Quilombola, 2007), citado por Walsh, Pedagogías decoloniales.., 37. 
Aquí, a propósito de esta significativa reflexión, se debe destacar que el grupo Hisula-UPTC lidera grandes esfuerzos para la recuperación de esos andamios mentales en las poblaciones afrodescendientes, como es el caso del Proyecto de Maestras africanas y afrodescendientes en Colombia, Brasil, Guatemala, Venezuela, Gabón, Guinea Ecuatorial. Siglo XX a XXI.

La paz requiere la construcción de andamios mentales para la paz, y eso significa que se necesita no solo de la expresión de una buena voluntad, sino de la construcción de un discurso pedagógico para la paz, que se decante de los andamios mentales históricamente silenciados por la cultura dominante. No resulta suficiente la invocación de la paz; sin abordar los andamios mentales de las poblaciones vulnerables, no podremos conectarnos con sus formas de ser y de sentir; ese es el reto que tenemos para poder lograr una paz emancipatoria: el rencuentro con sus raíces de resiliencia.

La categoría Geomental busca conectarnos con la cultura de resistencia, que se encuentra silenciada, pero, para eso, necesitamos fortalecer epistemológicamente esa visión. Acudimos a la tradición historiográfica de la Escuela de Annales para fundamentar el Estado del Arte que sirve de sustrato epistemológico al concepto Geomental. El mismo se incorporó en la Comunidad Científica con la tesis doctoral, ${ }^{49} \mathrm{y}$ se ha desarrollado en múltiples trabajos y artículos, hasta macerar el concepto que hoy presentamos aplicado a un contexto específico, en el marco del Postacuerdo. De manera que son más de 20 años madurando un concepto ${ }^{50}$ y hoy ya cuenta con la aceptación de la Comunidad Científica internacional. Y nos satisface que, al interior de las Pedagogías del Sur, se hubieran incorporado trabajos que siguen esta misma dirección.

Las Pedagogías Alternativas y la Pedagogía de la Resiliencia requieren del estudio de los andamios mentales de nuestra sociedad, pues en ellos se encuentran los elementos estructurales de la capacidad de superación y emancipación de nuestros pueblos. El estudio del tiempo estructural nos devela los valores

49 José Pascual Mora García, La Dama, el Cura y el Maestro en el siglo XIX (Mérida: Universidad de Los Andes, 2004. Y tiene su referencia inicial en la Tesis Doctoral en Historia, Historia de las Mentalidades y de la Educación, en la Vicaría Foránea de La Grita, en el Tiempo Histórico de la Diócesis de Mérida de Maracaibo, 2001.

50 José Pascual Mora-García, "La Historia de las Mentalidades: ¿Una Alternativa Metodológica para la Historia de la Educación en Venezuela?", en Libro de resúmenes, del IV Congreso Iberoamericano de Historia de la Educación Latinoamericana Santiago de Chile, 1998. 
geomentales de una sociedad, nos evidencian las cualidades de la resiliencia de esa sociedad. Los andamios mentales nos permiten indagar en la carga de nuestro inconsciente colectivo, porque la violencia se induce culturalmente y la escuela tiene una gran responsabilidad en ese proceso. La escuela, también, enseña la discriminación, la descalificación e infravaloración de la mujer, la intolerancia ante la diversidad y la dialéctica de la negación. Aprendimos, desde la tradición aristotélica, declarar la guerra al extranjero por nacer fuera de la polis y, en consecuencia, declararlo como esclavo por naturaleza. La inducción a la xenofobia, la discriminación étnica y la infravaloración, tiene una raíz griega; hoy la asumimos como una herencia en nuestros engramas cognitivos. El feminicidio se ha legitimado desde la cultura griega; Sófocles, en la Antígona, nos narra su tragedia, para darnos cuenta de la condición alieni juris de la mujer, en la cual se legitimaba la dominación. Hoy construimos leyes para castigar al feminicida; la Ley Rosa Elvira Celis (2015) ha demostrado ser insuficiente para frenar la ola de feminicidios en Colombia. Solo con la realización de una repedagogización social y escolar y derrota del paradigma patriarcal podremos soñar con una sociedad sostenible.

El estudio de los andamios geomentales es básico para pensar en una Pedagogía de la Resiliencia; implica el estudio de la vida privada, las actitudes, las creencias, la taumaturgia,${ }^{51}$ los estudios de los rituales mágicos ancestrales, la historia de la infancia,${ }^{52}$ el estudio del amor cortesano y romántico, pero, también, los enfoques emergentes, donde el amor es una práctica más que un sentimiento; el estudio de la tanatafobia y la comprensión de la muerte en las comunidades éticas como discursos emergentes; ${ }^{53}$ la historia de la violencia estructural, así como la historia de la violencia silenciada en la historiografía oficial; la historia de la mujer, pero no solo de la heroína o los símbolos socialmente reconocidos, sino la historia de la mujer que mira "desde abajo", desde las mujeres negadas por la ortodoxia de pensamiento; ${ }^{54}$ la historia de la fiesta como expresión de la catarsis colectiva o, como dijera Vovelle, la fiesta como expresión de los tiempos

\footnotetext{
$51 \quad$ Marc Bloch, Les rois thaumaturges (París: Gallimard. [1924] 1983).

52 Philippe Ariès, L'enfant et la vie familiale sous l'Ancien Régime, citado por Robert Mandrou, Francia en los siglos XVII y XVIII (Barcelona: Labor, 1973).

53 Philippe Ariès, La muerte en Occidente (Barcelona: Argos Vergara, 1982).

54 Evelyn Cherpak, "Las mujeres en la Independencia. Sus acciones y contribuciones", en Las Mujeres en la Historia de Colombia, Magdalena Velásquez Toro et al., comps. (Bogotá: Presencia, 1995).
} 
catárticos ${ }^{55}$ de la sociedad ${ }^{56} \mathrm{y}$, al mismo tiempo, también no solo el estudio de los tiempos apolíneos, sino de los dionisiacos, que incluyen aquellos que lindan con la patología social.

Cuando se atan cabos, descubrimos puntos de inflexión entre la historia de lo cotidiano y su impacto en la pedagogía oficial de la época; cómo la razón patriarcal se reproduce en la razón escolar. Es una historia de la pedagogía por/ para la paz que busca ver "desde abajo", ${ }^{57}$ por eso, más que preocuparse por los metarrelatos, ahonda en la historia de los individuos. Así, pues, al abordar el estudio de la mentalidad colectiva boyacense, se rastrea no la historia de los prohombres, sino la relacionada con las poblaciones vulnerables, para encontrar en su cultura de resistencia la fuente de la resiliencia. La mentalidad no se afianza a partir de los prototipos o prohombres en la historia, que se fraguan en la conjunción entre el más representativo y el último de los campesinos boyacenses. Estas ideas, bastardeadas en el seno de las mentalidades, las ha incorporado el inconsciente colectivo en los andamios mentales cundiboyacenses. La memoria es un instrumento del poder; apoderarse de la memoria es una de las máximas preocupaciones de las clases dominantes, aquellas que han dominado y dominan en las sociedades históricas.

\section{Conclusiones}

A modo de síntesis, la propuesta de las Pedagogías Alternativas como estrategia para recuperar los andamios mentales resilientes en el marco del Postacuerdo en la región cundiboyacense nos permite delinear estas conclusiones:

Las Pedagogías Alternativas deben estudiar por igual la práctica escolar y los andamios mentales de la sociedad. Las Pedagogías Alternativas deben vincular el saber académico con el "utillaje mental" a fin de salvar el sesgo de la intelectualización de la práctica escolar.

\footnotetext{
55 José Pascual Mora García, "Las corridas de toros en las fiestas de San Sebastián”, Heurística. No. 10 (2009).

56 Marcos González Pérez, "La idea de nación”, en Investigación interdisciplinaria. Urdimbres y tramas, Marcos González y José Eduardo Rueda, comps. (2a ed, Bogotá: Magisterio, 2005); Marcos González Pérez, comp., Fiesta y nación en Colombia (Bogotá: Magisterio, 1998b).

57 Eric Hobsbawn y Terence Ranger, eds., La invención de la tradición (Barcelona: Crítica, 2002).
} 
Las Pedagogías Alternativas deben preocuparse por estudiar no solo las ideas de los pro-hombres de la sociedad, sino estudiar por igual los andamios mentales entre el líder protagónico y el último de los hombres de los sectores vulnerables de cada comunidad.

Las Pedagogías de la Resiliencia deben deconstruir los paradigmas hegemónicos que implantó el paradigma patriarcal y promover el desarrollo de una mentalidad colectiva que apueste y respete la pedagogía de la diversidad.

Las Pedagogías Alternativas deben trascender la tendencia periodizante, porque los ritmos temporales nunca son iguales cuando se trata de la cultura, la economía, la política, y mucho menos las prácticas educativas. Es erróneo seguir manteniendo la idea de un universal educativo de todos los tiempos y para todos los hombres.

Las Pedagogías Alternativas deben vincular y estudiar la temporalidad entre la historia de larga duración y el tiempo coyuntural. Se trata aquí de complejizar el discurso temporal, no de simplificarlo; aprender a leer los tiempos discontinuos.

Y las Pedagogías Alternativas deben arqueologizar la mentalidad colectiva, porque allí reposan los imaginarios, el inconsciente colectivo, las costumbres y las tradiciones que son más fuertes, en el proceso socio-cultural, que la misma práctica educativa y pedagógica. El error metodológico que siempre se cometió fue separar estos procesos. 


\section{Referencias}

Ariès, Philippe. “L'enfant et la vie familiale sous l'Ancien Régime”. En: Francia en los siglos XVII y XVIII, Mandrou, Robert. Barcelona: Labor, 1973.

Ariès, Philippe. La Muerte en Occidente. Barcelona: Argos Vergara, 1982.

Bloch, Marc. La Historia rural francesa: caracteres originales. Buenos Aires: FCE, 1978.

Bolaños Motta, José Ignacio. "Aulas multiculturales y aulas de paz. Dos propuestas para un país en Postconflicto". Revista Historia de la Educación Latinoamericana. Vol. 20. No. 31 (2018): 83-100.

Boff, Leonardo. San Francisco de Asis: Ternura y vigor. Caracas: Ediciones Paulinas, 2002.

Cabra García, Heidy Lorena. "Barreras para la inclusión social de las personas con discapacidad, un escenario de derechos humanos". Heurística. No. 20 (2017): 127-137.

Cherpak, Evelyn. "Las Mujeres en la Independencia. Sus acciones y contribuciones". En: Las mujeres en la Historia de Colombia, compilado por Velásquez Toro, Magdalena et al. Bogotá: Presencia, 1995.

Delgado Martínez, Andrea. "Oportunidades laborales para excombatientes: percepciones de los generadores de empleo en el Quindío y Risaralda". Heurística., No. 20 (2017): 53-64.

Dussel, Enrique. "La introducción de la transformación de la filosofía de K.-O. Apel y la filosofía de la liberación. Reflexiones desde una perspectiva latinoamericana". En: Fundamentación de la ética y la filosofía de la liberación, Apel, Karl-Otto, et al. México: Siglo XXI editores, 1992. 45-104.

Dussel, Enrique. Filosofía de la cultura y la liberación. México: UACM, 2006. Esté, Arnaldo. Los maleducados: de la Escuela Básica a la Universidad. Caracas: UCV, 1986. 
Freire, Paulo. Pedagogía de la tolerancia. México: Crefal, 2006.

González Pérez, Marcos. "La idea de nación”. En: Investigación Interdisciplinaria. Urdimbres y Tramas, compilado por González Pérez, Marcos y Rueda, José Eduardo. Bogotá: Magisterio, 1998.

González Pérez, Marcos, comp. Fiesta y nación en Colombia. Bogotá: Magisterio, 1998.

Giarracca, Norma, comp. ¿Una nueva ruralidad en América Latina? Buenos Aires: Clacso, 2000.

Hobsbawn, Eric y Ranger, Terence, eds. La invención de la tradición. Barcelona: Crítica, 2002.

Holling, Crawford Stanley y Walker Brian H. (2003), "Resilience defined". En: Internet Encyclopaedia of Ecological Economics, editada por International Society of Ecological Economics. http://www.ecoeco.org/pdf/resilience. pdf

Le Goff, Jacques. El orden de la memoria. Barcelona: Paidós, 1991.

Manchego Bonilla, Nohora Isabel. "Ajustes razonables y derechos humanos: una perspectiva de equidad, dignidad y trascendentalidad en la inclusión educativa". Heurística. No. 20 (2017): 28-40.

Mora García, José Pascual y D' Yongh Sosa, Arnaldo. “Educación en Emergencias (EeE): Una realidad que promete desarrollo. Experiencias en Haití; México, caso Ayotzinapa". Heurística. No. 17 (2014): 15-33. http:// www. saber.ula.ve/ handle123456789/40105

Mora García, José Pascual. "Editorial”. Heurística. No. 20 (2017): 13-14. http://www.saber.ula.ve/bitstream/handle/123456789/44993/editorial. pdf? sequence $=1 \&$ isAllowed $=\mathrm{y}$

Mora García, José Pascual. "Propuesta interdisciplinaria de maestría en educación mención: cultura de paz, derechos humanos (DDHH) y educación en 
emergencia (eee) en la Universidad de Los Andes- Táchira, núcleo Dr. Pedro Rincón Gutiérrez". Heurística. No. 15 (2012): 172-189. http://www.saber. ula.ve/bitstream/handle/123456 789/37307/articulo15.pdf?sequence=1

Mora García, José Pascual. "La cultura de paz y la racionalidad dialógica gadameriana: una mirada sobre la filosofía de la paz en Rotary". Heurística. No. 15 (2012): 221-228. http://www.saber.ula.ve/bitstream/handle/123456789/3 7310/articulo18.pdf?sequence $=1$

Mora García, José Pascual. “In Memoriam: Guillermo Hoyos Vásquez: el filósofo de la paz, se nos fue". Heurística. No. 15 (2012): 326-327. http://www.saber. ula.ve/bitstream/handle/123456789/ 37319/articulo27.pdf?sequence=1 \&isAllowed $=\mathrm{y}$

Mora García, José Pascual. "Editorial”. Heurística. No. 17 (2014): 11-14. http:// www.saber.ula.ve/handle/123456 789/40089

Mora-García, José Pascual. "La Historia de las Mentalidades: ¿Una Alternativa Metodológica para la Historia de la Educación en Venezuela?" En: Libro de Resúmenes del IV Congreso Iberoamericano de Historia de la Educación Latinoamericana. Santiago de Chile: Universidad Católica, 1998.

Mora-García, José Pascual. "Leopoldo Zea, el filósofo de la negritud y el indigenismo". Revista de Ciencias Sociales de la Región Centroccidental. No. 9 (2004): 163-171.

Mora García, José Pascual. "La Pedagogía de la Resiliencia y los Andamios Geomentales", Conferencia Inaugural de la XI Cohorte del Doctorado de Ciencias de la Educación, RUDECOLOMBIA, Cade UPTC. Tunja, 24 de agosto de 2018.

Mora García, José Pascual. "Las Pedagogías de la Resiliencia, estudio de casos", Conferencia en la Cátedra Historia y Prospectiva de la Universidad Latinoamericana (HISULA Cade UPTC). Tunja, 26 de agosto de 2018.

Mora García, José Pascual. "El disenso como esencia de la libertad de expresión en la democracia, una mirada histórica sobre la fragilidad de la democracia 
en América Latina." Conferencia en el Congreso Internacional Derecho “Construcción Democrática Para La Paz", USTA, Tunja, 24, 25 y 26 agosto 2017.

Mora García, José Pascual. "Las pedagogías de la resiliencia y su impacto en la productividad académica", Conferencia en el Seminario de Comunidades Académicas" del Doctorado en Ciencias de la Educación, XI Cohorte, UPTC, Tunja, 21 de marzo de 2019.

Mora García, José Pascual. "Luis Fernando Valero Iglesias, y su experiencia académica en Colombia y El Salvador", Conferencia en el marco del Foro: Pedagogía y Migraciones, Semana Aniversario de la UPTC, octubre de 2018.

Mora García, José Pascual. "Para una Pedagogía de las Migraciones en Colombia y América Latina (Impacto de las Migraciones en la Internacionalización del Curriculum”, Capítulo de libro, en edición, Tunja: Ciefed-UPTC. 2019.

Mora García, José Pascual. "La Maestra Argelia Mercedes Laya López. Aportes al imaginario afrodescendiente venezolano (1926 - 1997)". Revista Historia de la educación latinoamericana. Vol. 18. No. 27 (2016): 13-34. DOI: 10.19053/01227238.5507

Mora García, José Pascual. "Isidora y el Calipso Afrovenezolano", Conferencia en el Coloquio de Maestras Afrodescendientes. Jamaica. Tunja: UPTC (En proceso de edición).

Mora García, José Pascual. "Pedagogía geomental en la construcción de nación, estudio de caso: la maestra afrodescendiente Luz Marina Castillo Vallecilla (1964- ), Barbacoas, Nariño, en el Pacífico colombiano", Ponencia presentada en IV Coloquio de Maestras Afrodescendientes en el marco del XII Congreso Internacional de la SHELA, Temuco, Chile, 17-19 de octubre de 2018.

Mora-García, José Pascual. "Las Políticas Públicas en la Formación del Profesorado en Venezuela: (1999-2011), una Mirada Crítica a las Políticas de Investigación". En: Formação e políticas públicas na educação: Profissão e condição docente, organizado por Jardilino, José Rubens Lima et al. Jundiaí: Paco Editorial, 2014. 41-66. 
Mora García, José Pacsual. "El curriculum en Venezuela: del eficientismo social de Rath Tyler a la postmodernidad (1970-1997)". En: Desarrollo del curriculum en América Latina, editado por Díaz Barriga, Ángel y Garduño, José María. Buenos Aires: Miño \& Dávila/UNAM, 2014. 269-320.

Mora García, José Pascual. "Las maestras rurales en el cantón de La Grita (Venezuela) y la formación en la escuela de niñas (siglo XIX)". En: La maestra rural en Iberoamérica. Historias de vida de maestras, Tomo VIII, en Colección Educadores Latinoamericanos y del Caribe, editado por Soto Arango, Diana Elvira Soto Arango et al. Tunja: HISULA/SHELA/Universidad Pablo de Olavide/Rudecolombia, 2015. 245-270.

Mora García, José Pascual; Soto Arango, Diana y Jardilino, José Rubens Lima, "La historia de la educación en América Latina: Contribución y aportes de la Sociedad de Historia de la Educación Latinoamericana (SHELA) (19942015)". Revista História da Educação / History Education Journal. Vol. 21. No. 51 (2017): 351- 375.

Mora García, José Pascual y Bernal Villate, Sandra. "La Revista Historia de la Educación Latinoamericana en los retos editoriales del siglo XXI". Inclusiones. Vol. 5. Fasc. 4 (2018): 36-61.

Mora García, José Pascual. "Luis Bigott y la contribución a la pedagogía contrahegemónica". Heurística. No. 19 (2016): 310-322. Conferencia enmarcada en el Proyecto de investigación: LA UPTC en los desafíos del siglo XXI, una prospectiva desde los estudiantes y docentes, sgi 165 del Grupo HISULA, UPTC. Coordinado por la Dra. Diana Soto Arango. VI Congreso internacional sobre historia y prospectiva de las universidades de Europa y América (CIHPUEA). Tunja, Boyacá, 10, 11 y 12 de octubre del 2016

Mora García, José Pascual. "Paradigma Historiográfico emergente en la revista de historia de la educación latinoamericana (Rhela)". Pueblo-Continente. Vol. 25. No. 1 (2014): 295-312.

Mora García, José Pascual. "Las Pedagogías de la Resiliencia, estudio de casos". Conferencia en la Cátedra Historia y Prospectiva de la Universidad Latinoamericana (HISULA Cade UPTC). Tunja, 26 de agosto de 2018. 
http://www.uptc.edu.co/export/sites/default/facultades/f_educacion/ doctorado/educacion/newsletter/doc/2018/newsletter_1_2018.pdf

Mora García, José Pascual. "Las corridas de toros en las fiestas de San Sebastián. (Aproximación a un imaginario festivo regional tachirense". Heurística. No. 10 (2009): 55-64. http://www.saber.ula.ve/bitstream/handle/123456 789/27026/articulo4.pdf?sequence $=1 \&$ isAllowed $=y$

Mora García, José Pascual. "Simón Rodríguez, precursor del pensamiento pedagógico decolonial", videoconferencia en el Doctorado de Educación, Universidad Federal de Ouro Preto, Brasil, realizada el 5 de junio de 2019. https://www.youtube.com/watch?v=10IVNJtX9W0

Olano Correa, Carmen. Espacios Amerindios, Mhuyscas y Chibchas de Boyacá, tierras de resguardo siglos XVI al XVIII. Bogotá: Centro desarrollo Ideas, 2010.

Oliveros Marín, Elena. "Relación entre derechos humanos y derechos lingüísticos en el pueblo indígena Kankuamo de la Sierra Nevada de Santa Marta". Heurística. No. 20 (2017). Tesis de Maestría en DDHH, UPTC.

Organización de las Naciones Unidas para la Alimentación y la Agricultura (FAO). "Colombia: Programa de resiliencia (2017-2020)", http://www.fao. org/emergencies/recursos/documentos/recursos-detalle/es/c/903122/

Otero, Marlene. "Retos de la Pedagogía en Postconflicto fronterizo. Docente de la ULA participa en jornada sobre formación de educadores en Iberoamérica". Heurística. No. 18 (2015): 272-274. http://epublica.saber.ula.ve/ index. $\mathrm{php} /$ heuristica/article/view/14836/21921925940

Otero, Marlene. "Trataron diversos conflictos en coloquio internacional sobre la paz, Coloquio internacional en paz, derechos humanos y movilidad fronteriza". http://prensa.ula.ve/2013/12/04/trataron-diversos-conflictosen-coloquio-internacional-sobre-la-paz

Otero, Marlene, "José Pascual Mora invoca la paz como emancipación”, La conferencia inaugural de la LXIV Convención Anual de AsoVAC. http://prensa. ula.ve/2014/11/20/jose-pascual-mora-invoca-la-paz-como-emancipacion 
Otero, Marlene. "Docentes del IEH (Instituto de Estudios Humanitarios) se manifiestan sobre proceso de paz de Colombia". http://prensa.ula.ve/2016/06/30/ docentes-del-ieh-se-manifiestan-sobre-proceso-de-paz-de-colombia

Otero, Marlene. "Guadalajara-México. Docentes de Shela y ULA Táchira manifestaron por desaparecidos de México". Heurística. No. 17, (2014): 315317. http://www.saber.ula.ve/bitstream/handle/123456789/40162/evento3. pdf? sequence $=1 \&$ isAllowed $=y$

Paternina Soto, Liliana. "El texto escolar sustentado en la resiliencia para poblaciones vulnerables. Fusagasugá 2008-2011”. Rhela. No. 16 (2011).

Pabón Triana, Marta. La recuperación de lenguas nativas como búsqueda de la identidad étnica. Bogotá: Universidad de los Andes, CCELA, 1995.

Periódico, Udenar. 1. "Con el maestro Hugo Zemelman en la Universidad de Nariño - Entrevista". Revista Historia de la Educación Colombiana. Vol. 17. No. 17 (2014): 19-27. http://revistas.udenar.edu.co/index.php/rhec/article/ view/2110.

Pérez Díaz, Omar et al. "IEH Nodo San CRISTÓBAL dictó videoconferencia invitado por la universidad privada Dr. Rafael Belloso Chacín en el Postdoctorado: estado, políticas públicas y paz". Heurística. No. 18 (2015): 268-269. http://www.saber.ula.ve/bitstream/handle/123456789/42050/ art21.pdf? sequence $=1 \&$ isAllowed $=y$

Rojas Quiñónez, Claudia. "La diversidad lingüística en Colombia: protección jurídica de las lenguas indígenas”, Pensamiento Jurídico. No. 22 (2008): 253-292.

Soto Arango, Diana y Pulido Huertas, Diana Carolina. "Las prácticas pedagógicas en Colombia en el marco del Postconflicto", Conferencia realizada por José Pascual Mora García, el 18 de mayo de 2018, en el Auditorio Gabriela Mistral de la Universidad Juan de Castellanos, organizada por el Sindicato de Maestros de Boyacá, Congreso de Pedagogía Interdepartamental. El Licenciado. No. 5 (mayo 2018). UPTC. http://www.uptc.edu.co/ export/sites/ default/facultades/f_educacion/licenciado/doc/2018/lic_5_2018.pdf 
Soto Arango, Diana Elvira; Mora García, José Pascual; Jardilino, José Rubens Lima. "Formación de docentes y modelo pedagógico en la Universidad Pedagógica y Tecnológica de Colombia". Revista Historia de la Educación latinoamericana. Vol. 19. No. 29 (2017): 35-66.

Suárez Ojeda, Elbio Néstor. "Introducción. Resiliencia y Subjetividad”. En: Resiliencia y Subjetividad. Los ciclos de la vida, compilado por Melillo, Aldo; Suárez Ojeda, Elbio Néstor y Rodríguez, Daniel. Buenos Aires: Paidós, 2004.

Suescún, Armando. "El Derecho Chibcha, siglo IX-siglo XVI". En: Derecho y Sociedad en la Historia de Colombia, Tomo I, $2^{\mathrm{a}}$ ed. Tunja: UPTC, 2012.

Trimiño Velásquez, Celina y Amézquita Aguirre, Luisa. "Reflexiones desde la universidad sobre educación en derechos humanos y para la paz". Revista Historia de la Educación Latinoamericana. Vol. 20. No. 31 (2018): 101124.

Walsh, Catherine. Pedagogías decoloniales, prácticas insurgentes de resistir, (re) existir, y (re) vivir. Tomo I. Buenos Aires: Clacso, 2013.

Zavala, Silvio Arturo. La filosofía de la conquista y otros textos. Caracas: Biblioteca Ayacucho, 2005.

Zea, Leopoldo. Dialéctica de la conciencia americana. México: UNAM, 1974.

Zemelman, Hugo y Quintar, Estela B. Conversaciones acerca de interculturalidad y conocimiento. México: IPN/IPECAL, 2007. 